\title{
miRNA-20a upregulates TAK1 and increases proliferation in osteosarcoma cells
}

\author{
Guangke Yuan ${ }^{1,2}$, Yanqing Zhao ${ }^{2}$, Dongjin Wu**,1, Chunzheng Gao*,1 \& Zhaode Jiao ${ }^{2}$ \\ ${ }^{1}$ Department of Orthopedics, The Second Hospital of Shandong University, Shandong University, NO 247, Beiyuan Street, Jinan \\ 250000, China \\ ${ }^{2}$ Department of Orthopedics, Yidu Central Hospital of Weifang, South Linglongshan Road, NO 4138, Weifang 262500, China \\ * Author for correspondence: chunzhenggao365@163.com \\ ** Author for correspondence: dongjinwu365@163.com
}

\begin{abstract}
Aim: The aim of this study is to explore the function of miR-20a in osteosarcoma. Materials \& methods: miR-20a expression was measured by real-time PCR. miR-20a mimics, inhibitor and scramble siRNA were transfected into osteosarcoma cells to observe effects on colony formation and tumor growth. Moreover, relationships of miR-20a with TAK1 were investigated by western blot and luciferase activity. Results: We found that miR-20a was downregulated in osteosarcoma, and overexpression of miR-20a reduced colony formation and tumor growth. Furthermore, the data revealed that the function of miR-20a was probably exerted via targeting the TAK1 expression. Overexpression of miR-20a sensitizes the osteosarcoma cells to chemotherapeutic drugs. Conclusion: Our data identify the role of miR-20a in osteosarcoma growth, indicating its potential application in chemotherapy.
\end{abstract}

First draft submitted: 25 September 2017; Accepted for publication: 16 November 2017; Published online: 12 January 2018

Keywords: apoptosis $\bullet$ cell proliferation $\bullet$ cisplatin $\bullet$ doxorubicin $\bullet$ drug resistance $\bullet$ miR-20a $\bullet$ NF-kB pathway $\bullet$ osteosarcoma • synergism • TAK1

Osteosarcoma is the most common primary malignant bone tumor with high morbidity in young adults and children, comprising $2.4 \%$ of all malignancies in pediatric patients and about $20 \%$ of all primary bone tumors [1]. It occurs mainly around regions with active bone growth and reparation. Osteosarcoma is highly aggressive and responded poorly to chemotherapy, and the 5-year survival rate for patients with metastatic osteosarcoma is only $14 \%$ [2]. Therefore, it is of extreme significance to elucidate novel molecular targets to develop alternative therapeutic target for this disease.

miRNAs are a small family of noncoding RNAs that play important roles in the development of human diseases by negatively regulating gene expression at either post-transcriptional or translational levels [3,4]. Over the last decade, many miRNAs have been showed in regulating diverse biological events such as cell proliferation, differentiation and apoptotic processes [5-7], which are important in the development of cancer. Accumulated evidence indicated that aberrant expression of miRNAs associates with various types of cancer. These dysregulated miRNAs function as either oncogenes or tumor suppressors in carcinogenesis and progression of cancers [8,9]. Thus, to explore the aberrant miRNAs expression in osteosarcoma might lead to the discovery of novel miRNAs biomarkers.

In this study, we found that miR-20a was downregulated in osteosarcoma samples and osteosarcoma cell lines, and the ectopic expression of miR-20a reduced cell colony formation in vitro and tumor growth in vivo. Furthermore, bioinformatic prediction and experimental validation revealed that the function of miR-20a was probably exerted via targeting the TAK1 expression. To translate these findings, overexpression of miR-20a sensitizes the osteosarcoma cells to chemotherapeutic drugs. Collectively, our data identify the key role of miR-20a in osteosarcoma growth, indicating its potential application in cancer chemotherapy.

Future Medicine 


\section{Materials \& methods}

\section{Cell culture}

Cell lines were purchased from the American Type Culture Collection (VA, USA) and cultured in DMEM with $10 \%$ fetal bovine serum at $37^{\circ} \mathrm{C}$ in $5 \% \mathrm{CO}_{2}$ cell culture incubator.

\section{Quantification of RNAs}

Total RNA was extracted from cells using TRIzol essentially as described previously [10]. Total RNA was extracted from cell cultures by using Purelink RNA mini Kit (ThermoFisher Scientific, NY, USA), following the manufacturer's instructions. $1 \mu \mathrm{g}$ of total RNA was reverse transcribed using qScript cDNA Supermix (Quantabio, MA, USA) to generate the first-strand cDNA. Quantitative real-time PCR were accomplished using SYBR-Green Master Mix (ThermoFisher Scientific). The relative gene expression was calculated using the comparative Ct method, which was normalized to the actin expression (endogenous control). The specificity of the PCR products was confirmed by analyzing the melting curve. All amplification reactions were performed in triplicate.

\section{Transfections}

All transfections were performed using Lipofectamine ${ }^{\circledR} 2000$ (ThermoFisher Scientific) according to the manufacturer's instructions. The miR-20a inhibitor or mimics and the scramble controls were obtained from GenePharma (Shanghai, China). The miR-20a inhibitor and mimics were used at a final concentration of $40 \mathrm{nM}$ [11].

\section{Immunoblotting}

Total cultured cells were harvested in radioimmunoprecipitation assay lysis buffer $(25 \mathrm{mM}$ Tris $\mathrm{pH} 7.4,150$ $\mathrm{mM} \mathrm{NaCl}, 5 \mathrm{mM}$ EDTA, $1 \%$ Triton-X, $1 \mu \mathrm{g} / \mathrm{ml}$ pepstatinA, $1 \mu \mathrm{g} \cdot \mathrm{ml}$ leupeptin, $1.5 \mu \mathrm{g} / \mathrm{ml}$ aprotinin, $0.1 \mathrm{mM}$ phenylmethylsulfonyl fluoride). Equal amounts of denatured protein lysates were resolved in the sodium dodecyl sulfate-polyacrylamide gels for electrophoresis and then transferred to polyvinylidene difluoride membranes. The membranes were blocked with 5\% nonfat milk in tris-buffered saline containing $0.1 \%$ Tween for $1 \mathrm{~h}$ at room temperature and subsequently probed with primary antibodies overnight at $4^{\circ} \mathrm{C}$. The blots were incubated with HRP-conjugated secondary antibodies for $1 \mathrm{~h}$ at room temperature and visualized using enhanced chemiluminescence on ChemiDoc XRS systems.

\section{Luciferase reporter assay}

For the luciferase reporter assay, HEK 293T cells were seeded in a 48-well plate and were grown to $70-80 \%$ confluence. To explore the interaction between miR-20a and 3' untranslated region (UTR) of TAK1, HEK 293T cells were cotransfected with $40 \mathrm{nM}$ of either scramble or miR-20a mimics and $100 \mathrm{ng}$ of either pmirGLOTAK1-3'UTR-WT or pmirGLO-TAK1-3'UTR-MUT using Lipofectamine 2000. The cells were harvested $48 \mathrm{~h}$ after transfection and analyzed using the Dual-Luciferase Reporter Assay System (Promega, WI, USA). A plasmid constitutively expressing renilla luciferase was cotransfected as an internal control to correct for differences in both transfection and harvesting efficiencies [12]. The transfections were performed in duplicate, and at least three independent experiments were performed [13].

\section{Immunofluorescence}

6- $\mu \mathrm{m}$-thick sections of samples were air-dried and fixed with ice-cold $4 \%$ paraformaldehyde for $15 \mathrm{~min}$, washed three-times with phosphate-buffered saline (PBS). Sections were permeabilized with $0.1 \%$ Triton X-100 in PBS for $15 \mathrm{~min}$ at room temperature. After washing three-times with PBS, the slides were blocked in PBS with 1\% BSA for $1 \mathrm{~h}$ at room temperature. Tissues were incubated with the Ki67 antibody overnight in a humidified chamber at $4^{\circ} \mathrm{C}$. Following three-times washing with PBS containing $1 \%$ Tween-20, the slides were incubated with fluorophore-conjugated antimouse/rabbit secondary antibodies and DAPI. Stained slides were observed with a Nikon Eclipse Ti confocal microscope (Nikon, Tokyo, Japan).

\section{Soft agar colony formation assay}

A standard two-layer soft agar culture was performed with a bottom layer of $0.6 \%$ agarose (Sigma Aldrich, MO, USA) and a top layer of $0.35 \%$ agarose. 500 cells per well were seeded with warm top agar in 6-well plates and incubated for 2 weeks, colonies were scored with the aid of a microscope [14]. 


\section{Synergism analysis}

5000 of G-63 or G-292 cells per well were plated into 96-well plate and incubated overnight and then treated with miR-20 inhibitor at the concentration of $5,10,15,20,25,30,35,40 \mathrm{nM}$, anticancer drugs (doxorubicin or cisplatin) at concentration of $0.95,1.9,3.75,7.5,15,30,60,120 \mathrm{nM}$, or combinations of miR-20a inhibitor and anticancer drug at constant ratios for $72 \mathrm{~h}$. Cell viability was determined by Alamar Blue vital dye that measures the activity of the mitochondrial respiratory chain.

\section{Tumor xenografts}

All the animal experimental procedures were performed according to the Shandong University ethical guidelines for animal experiments. $1 \times 10^{6}$ G-292 cells stably overexpressed miR-20a or vehicle in the $100 \mu \mathrm{l}$ PBS were mixed with $100 \mu \mathrm{l}$ Matrigel and subcutaneously injected into the right flank. Mice were sacrificed 30 days after tumor inoculation. The final tumor weights were measured. Primary tumors were resected and optimal cutting temperature embedded for further analysis.

\section{Statistical analyses}

All the statistical analyses were performed using GraphPad Prism. Student's t-test was carried out. All data were expressed as the means \pm standard error of the mean. In all cases, $\mathrm{p}$-values were calculated and $\mathrm{p}<0.05$ was considered statistically significant.

\section{Results}

\section{miR-20a is decreased in osteosarcoma tissues \& cell lines}

To evaluate the expression level of miR-20a in human osteosarcoma tissues, we analyzed the miRNA expression profile from a previously published dataset (gene expression omnibus accession GSE7828). As shown in Figure 1A $\& \mathrm{~B}$, miR-20a was obviously downregulated in human osteosarcoma tissues compared with the normal bone tissue samples ( $\mathrm{p}=0.0227$; Figure 1A \& B). The expression of miR-20a was also tested in G-292, Saos2, MG-63, HOS, U2OS and normal cell line hFOB using real-time PCR. The five osteosarcoma cell lines exhibited a markedly lower expression level of miR-20a compared with hFOB cells (Figure 1C). These data indicated that miR-20a might function as a potential tumor-suppressive role in osteosarcoma.

\section{miR-20a downregulates the expression of TAK1}

To explore the molecular mechanism of miR-20a in osteosarcoma, TargetScan (version 7.1) and (version 2010-1101) miRanda were used to predict the potential target. Bioinformatics analysis identified that TAK1 as potential target for miR-20a. The 3'UTR of TAK1 mRNA harbored sequences complementary to the miR-20a seed sequences (Figure 2A). Therefore, we examined TAK1 expression level after transfection with miR-20a mimics and found that miR-20a mimics inhibited TAK1 expression at the mRNA and protein level (Figure 2B \& C). The TAK1 mRNA level was increased when the cells were transfected with miR-20a inhibitor (Figure 2D). TAK1 was known to be physiologically involved in NF-kB pathway functioning as signal transducer. As expected, the phospho p65 and NF-kB activity was decreased in cells transfected with the mimics compared with the control cells (Figure 2E). Conversely, the NF-kB activity was enhanced in cells transfected with the miR-20a inhibitor (Figure 2E). To further validate TAK1 is the direct target of miR-20a, we performed the luciferase reporter assays in HEK293T cells combined with site-directed mutagenesis. For this purpose, we cloned 3'UTR of TAK1 into downstream of the dual-luciferase reporter vector and then transfected these vectors into cells. The relative luciferase activity was obviously reduced in the 3'UTR-wt group (Figure 2F). Mutations in the predicted miR-20a target sites abrogated the inhibition by miR-20a mimics (Figure 2F), indicating that TAK1 was direct targets of miR-20a. In conclusion, the results suggested that TAK1 is indeed novel direct target of miR-20a.

\section{Overexpression of miR-20a reduces the proliferation of osteosarcoma cells in vitro \& in vivo}

To further investigate the role of miR-20a on osteosarcoma growth in vitro, miR-20a was stably overexpressed or knockdown in osteosarcoma cell lines using a lentivirus plasmid. Cell proliferation was measured using anchorageindependent growth assay (soft agar assay). Overexpression of miR-20a in G-292, MG-63 and Saos2 cells significantly inhibited proliferation (Figure 3A). To further confirm the effects of miR-20a on tumor growth in vivo, G-292 cells stably overexpressing miR-20a or scrambles were injected into the flanks of nude mice. 30 days after inoculation, the mice were sacrificed, and the tumor tissues were striped and weighted. We found that average 


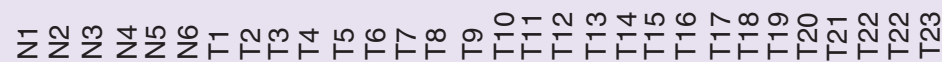

(A)

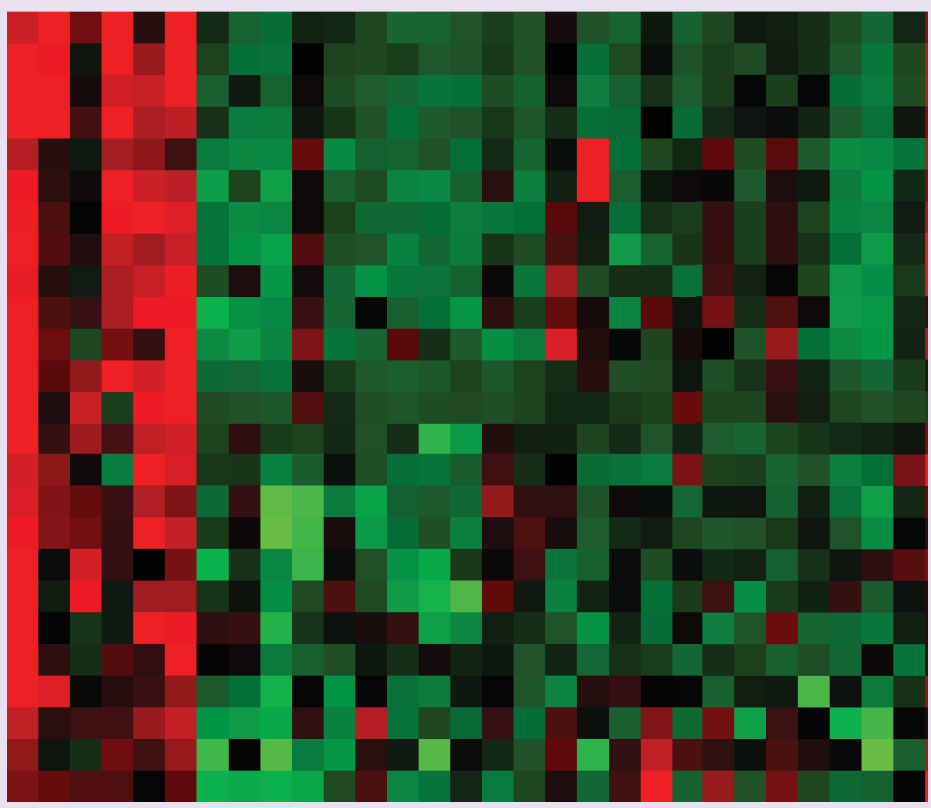

hsa-miR-123-3p

hsa-miR-123-5p

hsa-miR-126-3p

hsa-miR-218

hsa-miR-15a

hsa-miR-162

hsa-miR-162

hsa-miR-171

hsa-miR-16a

hsa-miR-17

hsa-miR-451

hsa-miR-142

hsa-miR-223

hsa-miR-582

hsa-miR-102

hsa-miR-105

hsa-miR-373

hsa-miR-340

hsa-miR-526a

hsa-miR-7

hsa-miR-551a

hsa-miR-641

hsa-miR-20a

hsa-miR-590

hsa-miR-768

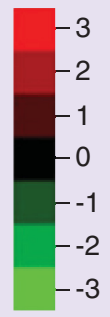

(B)

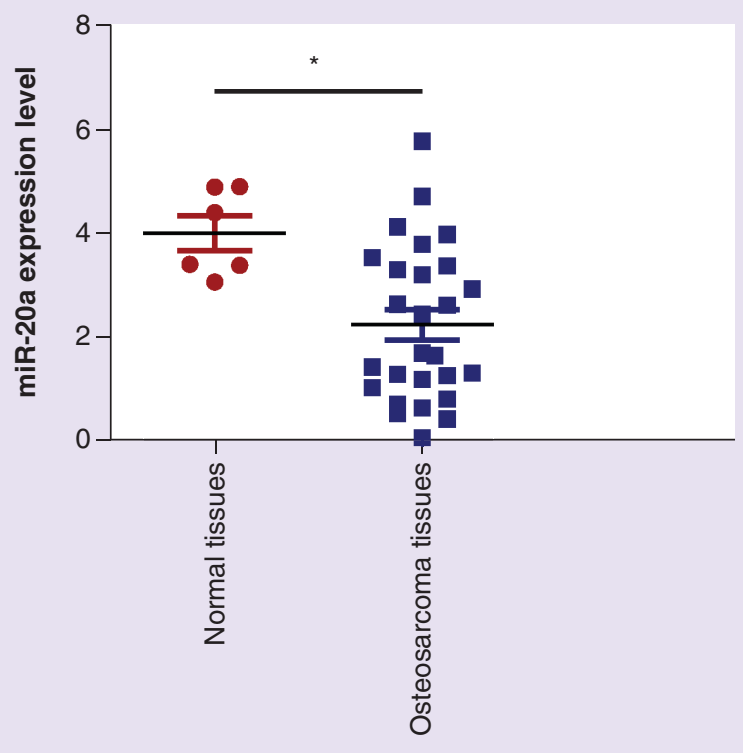

(C)

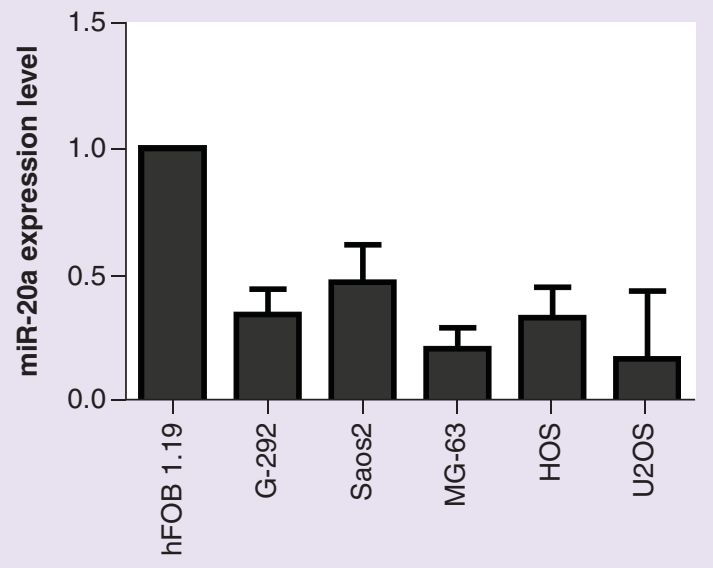

Figure 1. miR-20a is decreased in osteosarcoma. (A) Heatmap of selected miRNA expression in osteosarcoma tissues compared with selected miRNA expression in normal tissue. (B) Statistical analysis showed that miR-20a is significantly decreased in osteosarcoma tissues compared with normal tissues in miRNA expression assays ( $p$-value $=0.0227$ ). (C) Real-time PCR analysis of miR-20a expression level in osteosarcoma cancer cell lines compared with miR-20a content in normal cell line.

$* \mathrm{p}<0.05$.

N: Normal tissue; T: Tumor.

tumor weight was significantly decreased in miR-20a overexpressed group compared with the scramble (Figure 3B). In addition, we stained the tumor sections with the Ki67, a well-known proliferation marker. Consistently, the data showed that tumors overexpressed miR-20a exhibited less proliferative cells (Figure 3C). These data suggested that overexpression of miR-20a inhibited osteosarcoma cell growth in vitro and in vivo. 
(A)

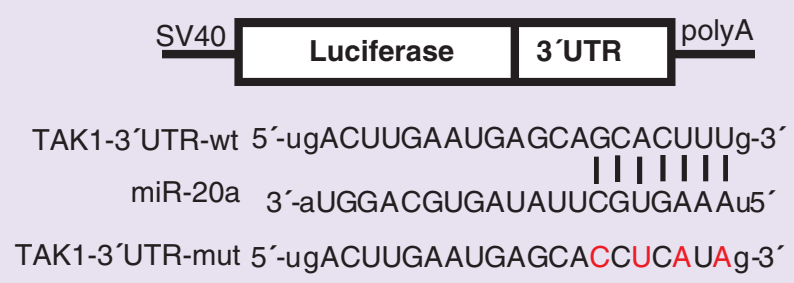

(C)

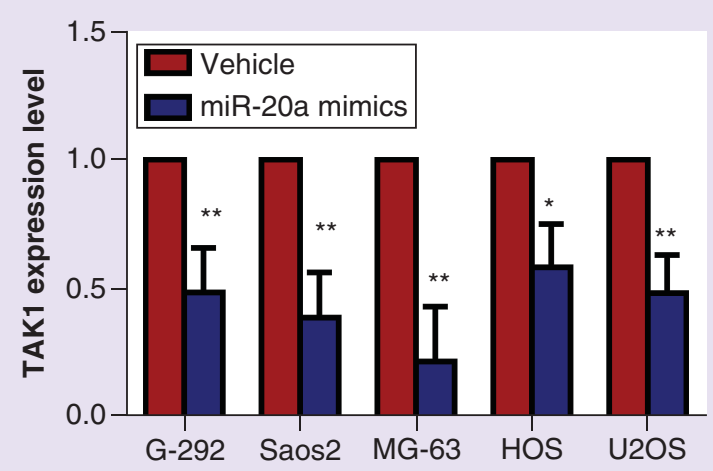

(E)

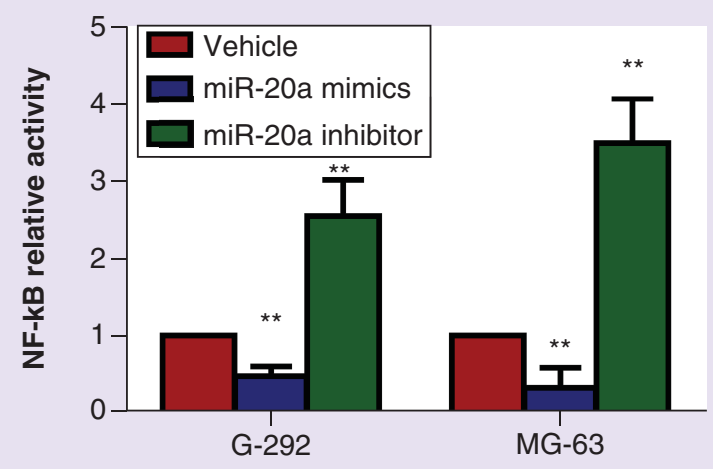

(B)

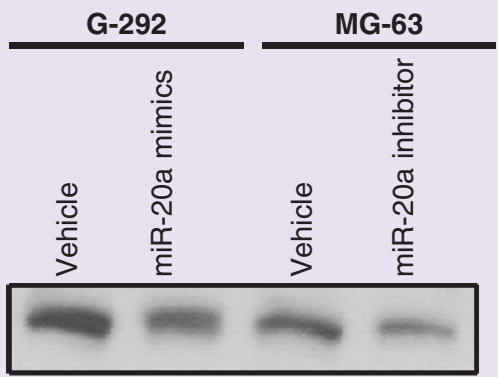

P-P65

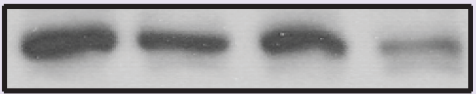

Total P65

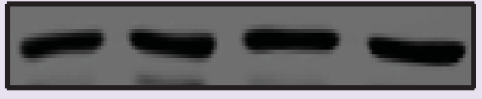

GAPDH

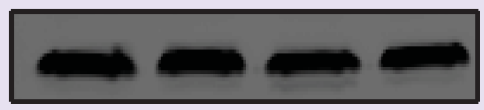

(D)

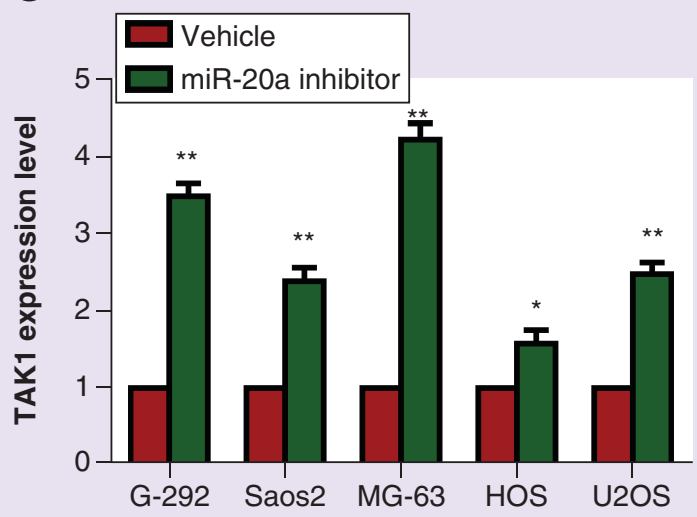

(F)

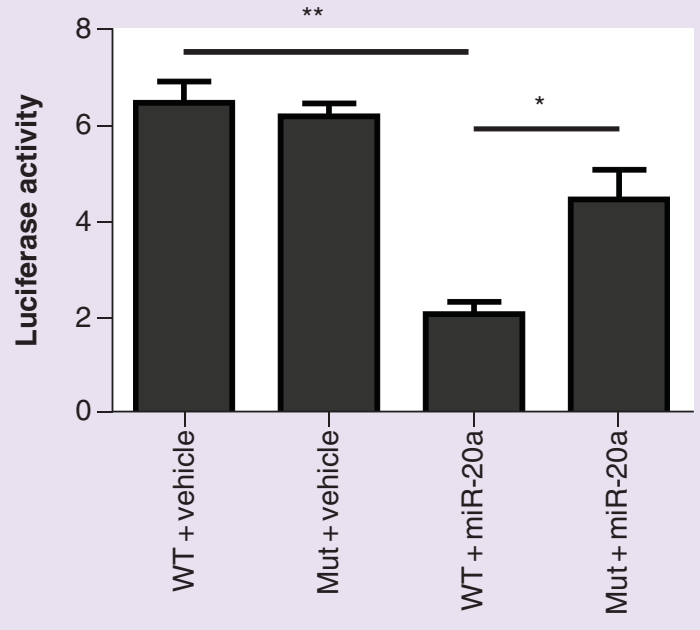

Figure 2. miR-20a downregulates the expression of TAK1. (A) The sequences in the 3'UTR of TAK1 are predicted to potentially bind to miR-20a. The red nucleotides are mutated to their complementary. (B) Western blot showed that overexpression of miR-20a with the mimics suppresses the expression of the TAK1 and phosphorylation of P65. (C) Real-time PCR showed that overexpression of miR-20a with the mimics reduces the TAK1 mRNA level in osteosarcoma cell lines. (D) Real-time PCR showed that knockdown of miR-20a with the inhibitor increases the TAK1 mRNA level in osteosarcoma cell lines. (E) Overexpression of miR-20a suppresses the NF-kB activity, knockdown of miR-20a enhances the NF-kB activity. (F) Dual-luciferase assays in HEK293T cells show that miR-20a suppresses luciferase activity in cells containing the 3'UTR wild-type plasmid, but not in the cells containing the 3'UTR mutant vector.

${ }^{*} \mathrm{p}<0.05$.

$* * \mathrm{p}<0.01$.

UTR: Untranslated region. 


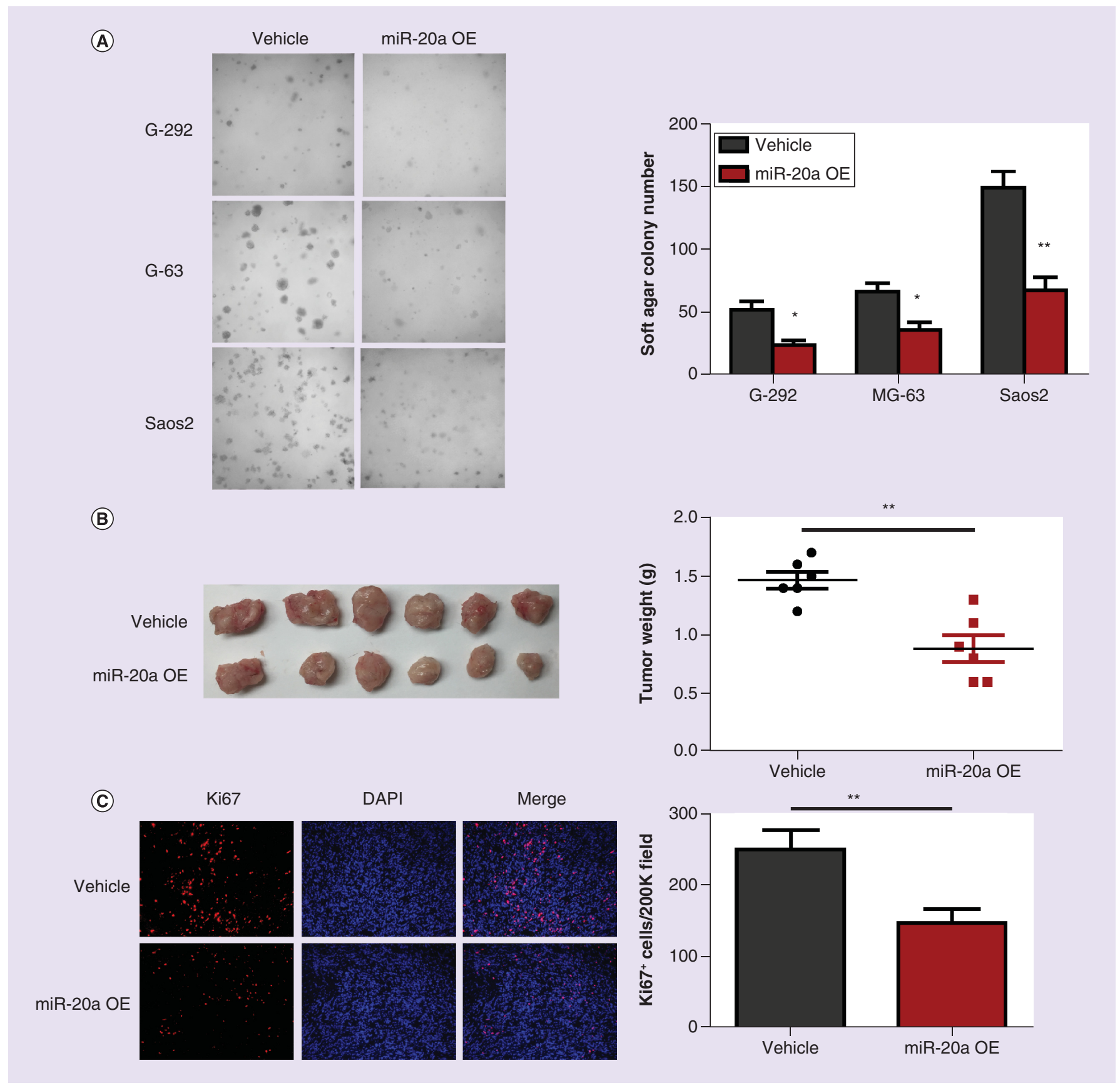

Figure 3. Overexpression of miR-20a reduces the proliferation of osteosarcoma cells. (A) Soft agar assay showed that the MG-63, G-292 and Saos2 cells stably overexpressing miR-20a formed fewer colonies compared with vehicle cells. (B) Overexpression of miR-20a in G-292 cells reduces the tumor growth in vivo. (C) Representative immunofluorescence staining images showing the decreased proliferation rate (Ki67/red, DAPI/blue) in tumors overexpressing miR-20a. 200K means 200 amplification.

$* \mathrm{p}<0.05$.

$* * p<0.01$.

miR-20a overexpression sensitizes osteosarcoma cells to the chemotherapy in vitro

The activation of NF-kB pathway is one of the major mechanism that was involved in the chemoresistance. To clarify the roles of miR-20a on the chemosensitivity in osteosarcoma cells, we explored the response to doxorubicin and cisplatin because the cell death induced by these two first-line drugs was closely associated with NF-kB activity. Doxorubicin or cisplatin at constant ratios was added at $24 \mathrm{~h}$ after transfection with miR-20a, the relative cell 
(A)

Doxorubicin

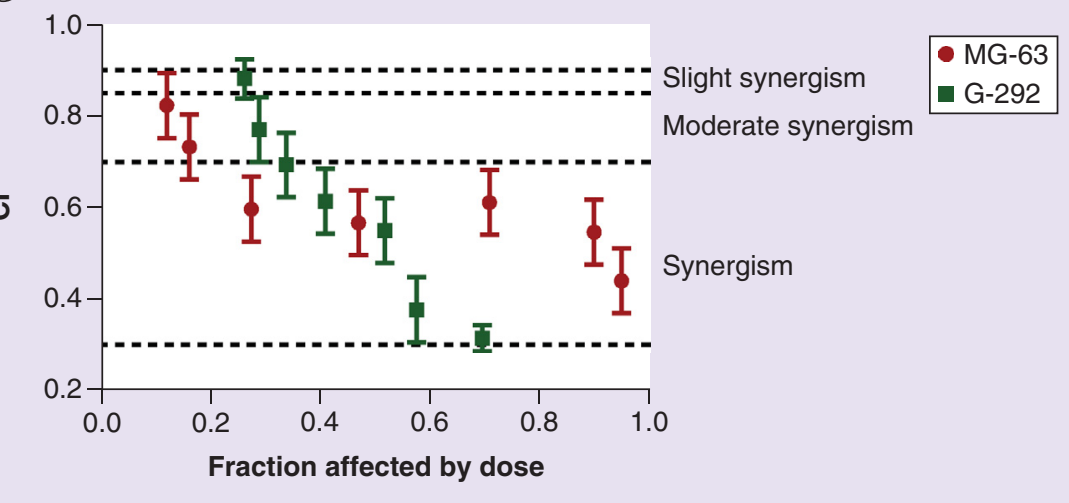

(B)

Cisplatin

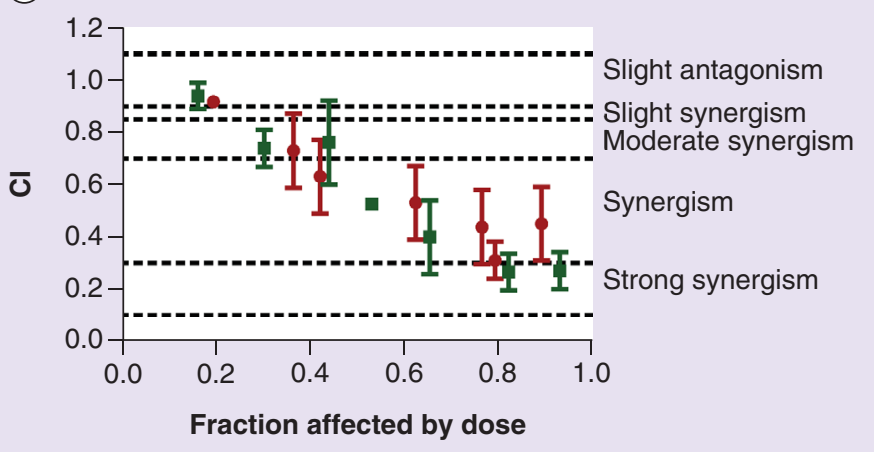

Figure 4. miR-20a overexpression sensitizes osteosarcoma cells to the chemotherapy in vitro. (A) Median effect analysis showed the interaction between miR-20a with doxorubicin in MG-63 and G-292 cell lines using CompuSyn (CompuSyn, Inc., Cambridge, UK) software. (B) Median effect analysis showed the interaction between miR-20a with cisplatin in MG-63 and G-292 cell lines using Compusyn software.

$\mathrm{Cl}$ : Combination index.

viability was measured with Alamar Blue assay at $72 \mathrm{~h}$. We performed synergism analysis and found various degrees of synergism between miR-20a mimics and doxorubicin or cisplatin (Figure $4 \mathrm{~A} \& \mathrm{~B}$ ) in these two cell lines. These findings indicated that overexpression of miR-20a sensitizes the osteosarcoma cells to chemotherapeutic agents.

\section{Discussion}

Osteosarcoma is the most common primary malignant bone tumor characterized by a highly aggressive clinical course in children and young adults [15]. Despite recent advances in therapeutic strategies, such as adjuvant chemotherapy, wide tumor excision and radiotherapy, the 5-year survival rate for patients with metastatic osteosarcoma is only $14 \%$ [16]. The underlying molecular genetic mechanisms involved in osteosarcoma remain poorly understood. The role of miRNAs in development of osteosarcoma remains unknown. A growing number of evidences have suggested that aberrant expression of miRNAs contributed to the carcinogenesis and progression of osteosarcoma. The importance of miRNAs in osteosarcoma and their potential utility as prognostic factors have become more and more clear [17]. In this study, we found that miR-20a expression is downregulated in osteosarcoma tissues compared with nontumor bone and in osteosarcoma cell lines. In addition, ectopic overexpression of miR-20a inhibited cell proliferation and tumor growth in vitro and in vivo, respectively. Furthermore, TAK1 was identified as a novel and direct target of miR-20a in osteosarcoma cells. To translate these findings, overexpression of miR-20a sensitizes the osteosarcoma cells to chemotherapeutic drugs. Collectively, our data identify the key role of miR-20a in osteosarcoma growth, indicating its potential application in cancer chemotherapy.

Here, we reported that miR-20a is downregulated in osteosarcoma and indicated that miR-20a is a tumorsupressor in osteosarcoma cell growth. miR-20a downregulation was reported in cutaneous squamous cell carcinoma, 
hepatocellular carcinoma and pancreatic cancer cells [18-20], whereas its upregulation was shown in uveal melanoma, colorectal cancer and cervical cancer [21-23]. These controversial data indicate that the function of miR-20a is possibly tumor specific and highly dependent on its downstream target in various cancers. Overexpression of miR-20a obviously inhibited colony formation in osteosarcoma cells and reduced tumor growth in vivo. These data suggested that miR-20a acted as tumor suppressor in osteosarcoma.

We further identified the possible direct targets of miR-20a in osteosarcoma cells. Among these potential target genes, we were interested in the TAK1 that is involved in the NF-KB pathway. The western blot and real-time PCR showed that overexpression of miR-20a significantly suppressed TAK1 expression at both mRNA and protein level. In addition, we showed that miR-20a directly bound to the $3^{\prime} \mathrm{UTR}$ of TAK1, which contains the miR-20a-binding sites. The activation of NF- $\mathrm{KB}$ pathway is one of the major mechanism that was involved in the chemoresistance. The synergism analysis showed various degrees of synergism between miR-20a mimics and doxorubicin or cisplatin in the two cell lines. These findings indicated that overexpression of miR-20a sensitizes the osteosarcoma cells to chemotherapeutic agents.

\section{Conclusion}

Our data report shows that miR-20a is decreased in osteosarcoma and identifies the key role of miR-20a in osteosarcoma growth in vitro and in vivo; indicating its potential application in chemotherapy.

\section{Future perspective}

Dysregulated microRNAs expression and their functions in tumorigenesis have gained much attention in recent years. Although miR-20a was shown to be involved in tumorigenesis, its function in osteosarcoma remains largely unknown. Although our study demonstrates the role of miR-20a in patients and osteosarcoma cells, it has many limitations. Hence, a large sample, prospective and multicenter clinical study should be conducted to evaluate the prognostic value of miR-20a in osteosarcoma patients. Furthermore, we expect to explore more accurate biomarkers to predict prognosis and guide our clinical treatment strategy formulation.

\section{Summary points}

- miR-20a was downregulated in human osteosarcoma samples.

- miR-20a was downregulated in osteosarcoma cell lines.

- The ectopic expression of miR-20a reduced cell colony formation in vitro.

- Overexpression of miR-20a decreases tumor growth in vivo.

- miRNA-20a regulates NF-kB activity.

- The function of miR-20a was probably exerted via targeting the TAK1 expression.

- miRNA-20a regulates TAK1 expression at mRNA level.

- Overexpression of miR-20a sensitizes the osteosarcoma cells to chemotherapeutic drugs.

Financial \& competing interests disclosure

The authors have no relevant affiliations or financial involvement with any organization or entity with a financial interest in or financial conflict with the subject matter or materials discussed in the manuscript. This includes employment, consultancies, honoraria, stock ownership or options, expert testimony, grants or patents received or pending, or royalties.

No writing assistance was utilized in the production of this manuscript.

Ethical conduct of research

The authors state that they have obtained appropriate institutional review board approval or have followed the principles outlined in the Declaration of Helsinki for all human or animal experimental investigations. In addition, for investigations involving human subjects, informed consent has been obtained from the participants involved. 


\section{References}

1. Yu W, Zhu J, Wang Y et al. A review and outlook in the treatment of osteosarcoma and other deep tumors with photodynamic therapy: from basic to deep. Oncotarget 8(24), 39833-39848 (2017).

2. Hutanu D, Popescu R, Stefanescu H et al. The molecular genetic expression as a novel biomarker in the evaluation and monitoring of patients with osteosarcoma-subtype bone cancer disease. Biochem. Genet. 55(4), 291-299 (2017).

3. Romano G, Veneziano D, Acunzo M, Croce CM. Small non-coding RNA and cancer. Carcinogenesis 38(5), 485-491 (2017).

4. Kim SW. The role of microRNAs in colorectal cancer. Korean J. Gastroenterol. 69(4), 206-211 (2017).

5. Zhang D, Wang Y, Shi Z et al. Metabolic reprogramming of cancer-associated fibroblasts by IDH3alpha downregulation. Cell Rep. 10(8), 1335-1348 (2015).

6. Wei Z, Cui L, Mei Z, Liu M, Zhang D. miR-181a mediates metabolic shift in colon cancer cells via the PTEN/AKT pathway. FEBS Lett. 588(9), 1773-1779 (2014).

7. Zhang D, Shi Z, Li M, Mi J. Hypoxia-induced miR-424 decreases tumor sensitivity to chemotherapy by inhibiting apoptosis. Cell Death Dis. 5, e1301 (2014).

8. Armand-Labit V, Pradines A. Circulating cell-free microRNAs as clinical cancer biomarkers. Biomol. Concepts 8(2), 61-81 (2017).

9. Vanacore D, Boccellino M, Rossetti S et al. MicroRNAs in prostate cancer: an overview. Oncotarget 8(30), 50240-50251 (2017).

10. Luo QL, Wang KP, Xiao K, Wang CG, Hu ZL. A rapid and high-quality method for total RNA isolation from Haematococcus pluvialis. Genet. Mol. Res. GMR 16(2), doi:10.4238/gmr16029614 (2017).

11. Nie S, Li K, Huang Y, Hu Q, Gao X, Jie S. miR-495 mediates metabolic shift in glioma cells via targeting Glut1. J. Craniofac. Surg. 26(2), e155-e158 (2015).

12. Lan J, Xue Y, Chen $\mathrm{H}$ et al. Hypoxia-induced miR-497 decreases glioma cell sensitivity to TMZ by inhibiting apoptosis. FEBS Lett. 588(18), 3333-3339 (2014).

13. Papageorgiou I, Court MH. Identification and validation of microRNAs directly regulating the UDP-glucuronosyltransferase $1 \mathrm{~A}$ subfamily enzymes by a functional genomics approach. Biochem. Pharmacol. 137, 93-106 (2017).

14. Yuan H, Wang Z, Gao C et al. BCR-ABL gene expression is required for its mutations in a novel KCL-22 cell culture model for acquired resistance of chronic myelogenous leukemia. J. Biol. Chem. 285(7), 5085-5096 (2010).

15. Yang Y, Yang R, Roth M et al. Genetically transforming human osteoblasts to sarcoma: development of an osteosarcoma model. Genes Cancer 8(1-2), 484-494 (2017).

16. Harrison DJ, Schwartz CL. Osteogenicsarcoma: systemic chemotherapy options for localized disease. Curr. Treat. Options Oncol. 18(4), 24 (2017).

17. Han Y, Liu M, Wang Z, Huang M, Xu N, Wu L. Serum microRNAs related with chemoradiotherapy resistance in advanced-stage cervical squamous cell carcinoma. Transl. Oncol. 10(3), 378-384 (2017).

18. Zhang L, Xiang P, Han X, Wu L, Li X, Xiong Z. Decreased expression of microRNA-20a promotes tumor progression and predicts poor prognosis of cutaneous squamous cell carcinoma. Int. J. Clin. Exp. Pathol. 8(9), 11446-11451 (2015).

19. Chen Y, Wang X, Cheng J et al. MicroRNA-20a-5p targets RUNX3 to regulate proliferation and migration of human hepatocellular cancer cells. Oncol. Rep. 36(6), 3379-3386 (2016).

20. Yuan W, Tang W, Xie Y et al. New combined microRNA and protein plasmatic biomarker panel for pancreatic cancer. Oncotarget 7(48), 80033-80045 (2016).

21. Achberger S, Aldrich W, Tubbs R, Crabb JW, Singh AD, Triozzi PL. Circulating immune cell and microRNA in patients with uveal melanoma developing metastatic disease. Mol. Immunol. 58(2), 182-186 (2014).

22. Huang G, Chen X, Cai Y, Wang X, Xing C. miR-20a-directed regulation of BID is associated with the TRAIL sensitivity in colorectal cancer. Oncol. Rep. 37(1), 571-578 (2017).

23. Xiong Y, Sun F, Dong P et al. iASPP induces EMT and cisplatin resistance in human cervical cancer through miR-20a-FBXL5/BTG3 signaling. J. Exp. Clin. Cancer Res. CR 36(1), 48 (2017). 
